\title{
Amiodarone in the COVID-19 Era: Treatment for Symptomatic Patients Only, or Drug to Prevent Infection?
}

\author{
Fabian Sanchis-Gomar ${ }^{1,2}$ (1) - Carl J. Lavie ${ }^{3} \cdot$ Daniel P. Morin $^{3} \cdot$ Carme Perez-Quilis $^{1}$ - Jari A. Laukkanen ${ }^{4,5}$. \\ Marco V. Perez ${ }^{2}$
}

Published online: 1 August 2020

(c) Springer Nature Switzerland AG 2020

\begin{abstract}
Amiodarone, one of the most widely prescribed antiarrhythmic drugs to treat both ventricular and supraventricular arrhythmias, has been identified as a candidate drug for use against the severe acute respiratory syndrome coronavirus 2 (SARSCoV-2). We present the rationale of using amiodarone in the COVID-19 scenario, as well as whether or not amiodarone administration represents a potential strategy to prevent SARS-CoV-2 infection, rather than simply used to treat patients already symptomatic and/or with severe coronavirus disease 2019 (COVID-19), based on current evidence.
\end{abstract}

Fabian Sanchis-Gomar

fabian.sanchis@uv.es

1 Department of Physiology, Faculty of Medicine, INCLIVA Biomedical Research Institute, University of Valencia, Av. Blasco Ibáñez, 15, 46010 Valencia, Spain

2 Division of Cardiovascular Medicine, Stanford University School of Medicine, Stanford, CA, USA

3 John Ochsner Heart and Vascular Institute, Ochsner Clinical School, The University of Queensland School of Medicine, New Orleans, LA, USA

4 Institute of Clinical Medicine, Department of Medicine, University of Eastern Finland, Kuopio, Finland

5 Department of Medicine, Central Finland Health Care District, Jyvaskyla, Finland

\section{Key Points}

Amiodarone has been identified as a candidate drug for use against the severe acute respiratory syndrome coronavirus 2 (SARS-CoV-2).

A multicenter study, the ReCOVery-SIRIO, is investigating the role of amiodarone in hospitalized patients with coronavirus disease 2019 (COVID-19) during the initial stages of infection.

Evidence on amiodarone use in COVID-19 patients is still scarce; we need to stay cautious and remain attentive for new results to obtain more robust conclusions.

While we wait for newly emerging evidence, both ventricular and supraventricular arrhythmias in COVID-19 patients should be treated with antiarrhythmic drugs in the same manner as patients who are not infected with COVID-19.

\section{Introduction}

In the urgent search for effective therapies against coronavirus disease 2019 (COVID-19), a wide range of candidates has emerged as potential drugs to be incorporated into the therapeutic armamentarium. In this regard, the endocytic pathway 
has been targeted as a therapeutic strategy in COVID-19 [1]. Several groups have identified amiodarone as a candidate drug for use against the severe acute respiratory syndrome coronavirus 2 (SARS-CoV-2) [2, 3]. Some have recommended administration of prophylactic intravenous amiodarone to mitigate the risk of sudden cardiac death (SCD) among patients infected with the novel SARS-CoV-2 [4]. A randomized study called ReCOVery-SIRIO has been started to evaluate the effects of amiodarone compared with usual care in symptomatic patients hospitalized with confirmed COVID-19 infection (ClinicalTrials.gov identifier: NCT04351763).

Amiodarone is among the most widely prescribed antiarrhythmic drugs to treat both ventricular and supraventricular arrhythmias, while also being important for maintaining sinus rhythm in patients with atrial fibrillation (AF) [5]. Although it does not decrease all-cause mortality in the primary prevention population, amiodarone is the most effective antiarrhythmic drug to prevent SCD [5]. Importantly, amiodarone reduces automatic implantable cardioverter defibrillator (ICD) shocks, being a first relatively easily available treatment option in patients experiencing electrical storm and repeated ventricular arrhythmias leading to recurrent ICD shocks [6]. In addition, amiodarone possesses anti-inflammatory and antioxidant properties [7, 8], i.e. pleiotropic effects, which can indirectly suppress ventricular arrhythmias [5]. However, amiodarone is not free of secondary adverse effects, contraindications and interactions with other drugs, including the potential to cause pulmonary toxicity and fibrosis, thyroid disease, hepatic toxicity, increased creatine levels, QT interval prolongation, and bradyarrhythmia [9]. Most of these adverse effects are more likely with long-term use of amiodarone. COVID-19 seems to be associated with higher rates of arrhythmia, either pre-existing or secondary to the disease $[10,11]$. COVID-19 patients are at risk of developing acute respiratory distress syndrome (ARDS), which makes amiodarone less attractive, but the sickest patients with viral infection have limited antiarrhythmic options. Thus, one question arises: can we use amiodarone to treat arrhythmias in COVID-19 patients? Arrhythmias, particularly AF, are common in COVID-19 patients, and amiodarone may be an effective and additive antiarrhythmic drug in these patients. We present here the rationale for amiodarone use in the COVID-19 scenario, as well as whether or not amiodarone administration represents a potential strategy to prevent SARS-CoV-2 infection, rather than simply used to treat patients already symptomatic and/or with severe COVID-19, based on current evidence.

\section{Rationale for Amiodarone Use}

\subsection{Pros}

\subsubsection{Infection Prevention or Treatment with Amiodarone Administration}

There is evidence that cationic amphiphilic drugs should be studied for possible interference with the life cycle of positive-stranded RNA viruses [12]. Specifically, the cationic amphiphilic drug amiodarone and its major metabolite $N$-desethylamiodarone $(N$-DEA) inhibit the entry of filoviruses (a family that includes Ebola virus) at similar concentrations to those found in the blood of patients treated for arrhythmias [13]. Amiodarone can interfere with SARS-CoV entry and amplification, and is able to block the spread of SARS-CoV in cell cultures by inhibiting infection at a postendosomal level. It accomplishes this without modifying the density of angiotensin-converting enzyme (ACE)-2 receptors on the cell surface or interfering with the attachment of SARS-CoV to the cells [14]. Thus, amiodarone has been described as an inhibitor of SARS-CoV spread through its ability to interfere with the endocytic pathway and by blocking ion channels, even when SARS-CoV could deliver its genome into the cytoplasm through the plasma membrane, thus bypassing the endocytic compartment [14]. Recently, Aimo et al. [15] proposed amiodarone as a candidate treatment of COVID-19 infection, either alone or as part of a combination of drugs. The authors also speculated that amiodarone administration is more effective in the setting of low viral loads, suggesting that amiodarone should be evaluated for use in previously exposed but asymptomatic individuals. ReCOVery-SIRIO is a multicenter effort investigating the role of amiodarone in hospitalized patients with COVID19 during the initial stages of infection to target virus replication by ion channel inhibition. This effect might be instrumental to attenuate virus entrance and replication before the cytokine storm caused by host immune response (Fig. 1).

\subsubsection{Anti-Inflammatory and Antioxidant Effects}

COVID-19 is associated with a high inflammatory burden [16], which may lead to vascular inflammation, myocarditis, and cardiac arrhythmias. It has been suggested that amiodarone administration prevents cytokine production and oxidative stress, which may protect lung tissue during the sepsis cascade. Particularly, SARS-CoV-2 infection triggers the activation of dendritic cells and cells of the monocyte/macrophage lineage, which activates the 




Fig. 1 Central illustration presenting the rationale for amiodarone use in COVID-19 patients: pros and cons. $A L I$ acute lung injury, $A R D S$ acute respiratory distress syndrome, BZPs benzodiazepines, COVID19 coronavirus disease 2019, CRP C-reactive protein, $G R$ glutathione reductase, GSH glutathione, $H C Q$ hydroxychloroquine, $H I V$ human immunodeficiency virus, $I L$ interleukin, $M C P-1$ monocyte chemoattractant protein 1, SARS-CoV-2 severe acute respiratory syndrome coronavirus 2, $S C D$ sudden cardiac death, $S O D$ superoxide dismutase, $T N F$ tumor necrosis factor

levels at baseline and at 1 and 2 years in 40 New York Heart Association (NYHA) class III patients treated with placebo and 40 NYHA class III patients treated with amiodarone. No changes were found in patients with non-ischemic cardiomyopathy, while amiodarone use increased TNF $\alpha$ levels in patients with ischemic cardiomyopathy at 1 year but not at 2 years, which suggests different effects of amiodarone depending on the duration of administration. In monocytes of patients with idiopathic dilated cardiomyopathy (DCM), Hirasawa et al. [20] found that amiodarone administration for 4 weeks attenuated monocyte cytokine and chemokine production in response to C-reactive protein (CRP), particularly decreasing both TNF $\alpha$ and MCP-1. These authors speculated that amiodarone might improve cardiac function through its anti-inflammatory effects, although this has not yet been proven in living humans. Regarding the mechanism involved in the reduction of certain cytokines, such as IL-6 or TNF $\alpha$, it has been hypothesized that amiodarone reduces their production by modulating the voltage-gated potassium channel, which regulates the activation of $\mathrm{T}$ lymphocytes [18], although further investigation is needed to clarify the specific mechanisms.

On the other hand, oxidative stress also plays a significant role in the pathophysiology of ischemic heart disease and heart failure, therefore improvements in antioxidant capacity might be beneficial in the treatment of these patients. Halici et al. [21] studied the effects of amiodarone 
administration on glutathione levels and the activity of superoxide dismutase, catalase, glutathione $S$-transferase, glutathione reductase and myeloperoxidase, in rats with carrageenan-induced paw edema. In this investigation, amiodarone reduced carrageenan-induced paw edema, relieved increases in the activities of catalase and glutathione $S$-transferase enzymes, minimized decreases in glutathione levels and the activities of superoxide dismutase and glutathione reductase enzymes, and amplified myeloperoxidase activity associated with paw edema. These results suggest that the anti-inflammatory activity of amiodarone may be related to its antioxidative effects. Ide et al. [22] determined the scavenging effects of amiodarone on oxygen free radicals, and its protective effects against oxygen radical-mediated injury in cardiac myocytes. Amiodarone decreased the intensity of the 5,5-dimethyl pyrroline- $\mathrm{N}$-oxide (DMPO)-OH signals in a dose-dependent manner, whereas disopyramide and atenolol had no such effects. Amiodarone also protected intact adult canine cardiac myocytes against $\cdot \mathrm{OH}$-mediated myocyte injury at a concentration of $10 \mathrm{mmol} / \mathrm{L}$, which suggests that amiodarone can protect cardiac myocytes against oxidative stress-mediated injury by directly scavenging oxygen free radicals [22].

Therefore, although the precise molecular mechanism by which amiodarone exerts an anti-inflammatory and/or antioxidant effect remains unclear, amiodarone-induced inhibition of cytokine and chemokine production and antioxidant capacity improvement may be critical factors mediating potential amiodarone-positive effects on COVID-19 therapy [23].

\subsection{Cons}

The use of amiodarone has significant limitations. In brief, during the early months of the pandemic, the combination of hydroxychloroquine and azithromycin was widely recommended to treat COVID-19; however, this treatment remains highly controversial in terms of efficacy and may prolong QTc. Amiodarone might aggravate this QT prolongation, which is associated with an increased risk of SCD [24].

Furthermore, the clinical application of amiodarone is limited by its marked extracardiac toxicity. Amiodarone has been associated with hepatic transaminitis, thyroid dysfunction, and pulmonary toxicity, although these adverse effects are directly related to the duration of treatment (amiodarone toxicity is cumulative and dose-dependent) [25]. Amiodarone's iodine moieties and extreme lipophilic character importantly contribute to its toxicity. Amiodarone-induced pulmonary toxicity can occur following acute or chronic treatment and is characterized by acute pulmonary hypersensitivity with patchy infiltrates or chronic pulmonary interstitial fibrosis, respectively. It is important to keep in mind that amiodarone-induced pulmonary fibrosis is a rare event that typically occurs after months to years of continued drug administration. It has also been shown that prehospital use of amiodarone may independently increase the risk for ARDS in patients with predisposing conditions for acute lung injury (ALI) [26]. Contributing factors to amiodaroneinduced pulmonary toxicity include its direct cytotoxic effects (principally mediated by $N$-DEA), phospholipidosis, immunological mechanisms, and activation of the angiotensin system; however, the precise mechanisms contributing to amiodarone-induced pulmonary toxicity remain incompletely understood. Importantly, pulmonary toxicity is more common in older people, increasing threefold every 10 years of age in patients aged over 60 years. Moreover, amiodarone affects metabolism, increasing circulating levels of other drugs such as warfarin, digoxin, non-steroidal antiinflammatory drugs, statins and benzodiazepines. Amiodarone also interacts with many antiretroviral medications used for the treatment of HIV infection. Finally, dronedarone, an amiodarone derivate, has also been identified as an active inhibitor of SARS-CoV-2 virus among a panel of US FDAapproved drugs screened to identify antiviral drug candidates for COVID-19 treatment [27]. Because dronedarone is less lipophilic than amiodarone, it has a smaller volume of distribution and a shorter elimination half-life, which ultimately reduces its toxicity. These features make dronedarone a worthwhile candidate to be considered.

\section{Conclusions and Future Perspectives}

First, despite the positive effects mentioned above, it should be noted that evidence on amiodarone use in COVID-19 patients is still scarce; we need to stay cautious and remain attentive to the expected results of the ongoing clinical trial in order to obtain more robust conclusions. That being said, the safety/risk profile of amiodarone is well known and it appears to be another candidate for clinical trials in the current COVID-19 scenario. A short-term use of amiodarone is relatively well tolerated. It may be speculated that amiodarone is more efficient with low viral loads and at the early stages of the COVID-19 infection. Accordingly, clinical trials of amiodarone should be mainly performed in individuals who are currently asymptomatic or with early disease, rather than severely symptomatic patients with severe COVID-19 infection. In the former, amiodarone should be better evaluated as an adjuvant drug. We must keep in mind that COVID-19 is characterized by ALI [9], and this could potentially be aggravated by long-term amiodarone administration. Therefore, shorter-term intervention trials with amiodarone could be preferred in the first stages of future studies. Moreover, in our opinion, retrospective and prospective studies in asymptomatic individuals previously exposed to SARS-CoV-2, as well as the incidence of 
infection between the present and prior users of amiodarone, are recommended.

While we wait for newly emerging evidence, arrhythmias in COVID-19 patients should be treated with antiarrhythmic drugs in the same manner as patients who are not infected with COVID-19. Arrhythmias, particularly AF, are common in COVID-19 and are associated with higher risk for morbidity and mortality. Amiodarone may be a particularly effective antiarrhythmic agent for these hospitalized patients who are prone to supraventricular and ventricular arrhythmias, although its safety and efficacy have to be better studied before wider-scale clinical recommendations.

Acknowledgements Fabian Sanchis-Gomar is supported by a postdoctoral contract granted by 'Subprograma Atracció de Talent-Contractes Postdoctorals de la Universitat de València'.

Availability of data and materials Not applicable.

Code availability Not applicable.

\section{Declarations}

Funding No external funding was used in the preparation of this manuscript.

Conflict of interest Fabian Sanchis-Gomar, Carl J. Lavie, Daniel P. Morin, Carme Perez-Quilis, Jari A. Laukkanen, and Marco V. Perez declare they have no potential conflicts of interest that might be relevant to the contents of this manuscript.

Ethics approval Not applicable.

Consent to participate Not applicable.

Consent for publication Not applicable.

\section{References}

1. Yang N, Shen HM. Targeting the endocytic pathway and autophagy process as a novel therapeutic strategy in COVID-19. Int J Biol Sci. 2020;16:1724-31.

2. Andersen PI, Ianevski A, Lysvand H, Vitkauskiene A, Oksenych $\mathrm{V}$, Bjoras M, et al. Discovery and development of safe-in-man broad-spectrum antiviral agents. Int J Infect Dis. 2020;93:268-76.

3. Kadioglu O, Saeed M, Johannes Greten H, Efferth T. Identification of novel compounds against three targets of SARS CoV-2 coronavirus by combined virtual screening and supervised machine learning. Bull World Health Organ. 2020. https://doi.org/10.2471/ BLT.20.255943.

4. Rali AS, Sauer AJ. COVID-19 pandemic and cardiovascular disease. US Cardiol Rev. 2020;14:e01.

5. Heijman J, Dobrev D. Pleiotropic actions of amiodarone: still puzzling after half a century. Naunyn Schmiedebergs Arch Pharmacol. 2013;386:571-4.

6. Gehi AK, Mehta D, Gomes JA. Evaluation and management of patients after implantable cardioverter-defibrillator shock. JAMA. 2006;296:2839-47.
7. Polat B, Cadirci E, Halici Z, Bayir Y, Unal D, Bilgin BC, et al. The protective effect of amiodarone in lung tissue of cecal ligation and puncture-induced septic rats: a perspective from inflammatory cytokine release and oxidative stress. Naunyn Schmiedebergs Arch Pharmacol. 2013;386:635-43.

8. Matsumori A, Ono K, Nishio R, Nose Y, Sasayama S. Amiodarone inhibits production of tumor necrosis factor-alpha by human mononuclear cells: a possible mechanism for its effect in heart failure. Circulation. 1997;96:1386-9.

9. Wong AW, Fidler L, Marcoux V, Johannson KA, Assayag D, Fisher $\mathrm{JH}$, et al. Practical considerations for the diagnosis and treatment of fibrotic interstitial lung disease during the COVID19 pandemic. Chest. 2020. https://doi.org/10.1016/j.chest .2020.04.019.

10. Kochav SM, Coromilas E, Nalbandian A, Ranard LS, Gupta A, Chung MK, et al. Cardiac Arrhythmias in COVID-19 Infection. Circ Arrhythm Electrophysiol. 2020;13:e008719.

11. Carpenter A, Chambers OJ, El Harchi A, Bond R, Hanington O, Harmer SC, et al. COVID-19 management and arrhythmia: risks and challenges for clinicians treating patients affected by SARSCoV-2. Front Cardiovasc Med. 2020;7:85.

12. Salata C, Munegato D, Piccoli E, Calistri A, Parolin C, Mirazimi A, et al. Amiodarone increases positive-strand RNA virus replication in vitro: implications for its use in patients with viral infections. J Antimicrob Chemother. 2016;71:280-1.

13. Salata C, Baritussio A, Munegato D, Calistri A, Ha HR, Bigler $\mathrm{L}$, et al. Amiodarone and metabolite MDEA inhibit Ebola virus infection by interfering with the viral entry process. Pathog Dis. 2015;73:ftv032.

14. Stadler K, Ha HR, Ciminale V, Spirli C, Saletti G, Schiavon M, et al. Amiodarone alters late endosomes and inhibits SARS coronavirus infection at a post-endosomal level. Am J Respir Cell Mol Biol. 2008;39:142-9.

15. Aimo A, Baritussio A, Emdin M, Tascini C. Amiodarone as a possible therapy for coronavirus infection. Eur J Prev Cardiol. 2020. https://doi.org/10.1177/2047487320919233.

16. Megens RT, Vijayan S, Lievens D, Doring Y, van Zandvoort MA, Grommes J, et al. Presence of luminal neutrophil extracellular traps in atherosclerosis. Thromb Haemost. 2012;107:597-8.

17. Lippi G, Sanchis-Gomar F, Henry BM. COVID-19: unravelling the clinical progression of nature's virtually perfect biological weapon. Ann Transl Med. 2020;8:693.

18. Ito H, Ono K, Nishio R, Sasayama S, Matsumori A. Amiodarone inhibits interleukin 6 production and attenuates myocardial injury induced by viral myocarditis in mice. Cytokine. 2002;17:197-202.

19. Oral H, Fisher SG, Fay WP, Singh SN, Fletcher RD, Morady F. Effects of amiodarone on tumor necrosis factor-alpha levels in congestive heart failure secondary to ischemic or idiopathic dilated cardiomyopathy. Am J Cardiol. 1999;83:388-91.

20. Hirasawa Y, Nakagomi A, Kobayashi Y, Katoh T, Mizuno K. Short-term amiodarone treatment attenuates the production of monocyte cytokines and chemokines by C-reactive protein and improves cardiac function in patients with idiopathic dilated cardiomyopathy and ventricular tachycardia. Circ J. 2009;73:639-46.

21. Halici Z, Dengiz GO, Odabasoglu F, Suleyman H, Cadirci E, Halici M. Amiodarone has anti-inflammatory and anti-oxidative properties: an experimental study in rats with carrageenan-induced paw edema. Eur J Pharmacol. 2007;566:215-21.

22. Ide T, Tsutsui H, Kinugawa S, Utsumi H, Takeshita A. Amiodarone protects cardiac myocytes against oxidative injury by its free radical scavenging action. Circulation. 1999;100:690-2.

23. Nakaya $H$. Does anti-inflammatory action of amiodarone explain the high efficacy in patients with heart failure? Circ J. 2009;73:622-3.

24. Chorin E, Dai M, Shulman E, Wadhwani L, Bar Cohen R, Barbhaiya $\mathrm{C}$, et al. The QT interval in patients with SARS-CoV-2 
infection treated with hydroxychloroquine/azithromycin. medRxiv. 2020. https://doi.org/10.1101/2020.04.02.20047050.

25. Hamilton D Sr, Nandkeolyar S, Lan H, Desai P, Evans J, Hauschild $\mathrm{C}$, et al. Amiodarone: a comprehensive guide for clinicians. Am J Cardiovasc Drugs. 2020. https://doi.org/10.1007/s4025 6-020-00401-5.

26. Karnatovskaia LV, Festic E, Gajic O, Carter RE, Lee AS, Illness USC, et al. Prehospital amiodarone may increase the incidence of acute respiratory distress syndrome among patients at risk. J Crit Care. 2012;27:447-53.

27. Jeon S, Ko M, Lee J, Choi I, Byun SY, Park S, et al. Identification of antiviral drug candidates against SARS-CoV-2 from FDA-approved drugs. Antimicrob Agents Chemother. 2020;64(7):e00819-e820. https://doi.org/10.1128/AAC.00819-20. 